\title{
PRESENTACIÓN Y RESULTADOS PRELIMINARES DE LA BATERÍA NOMBELA (I). UN NUEVO INSTRUMENTO PARA EVALUAR EL DETERIORO SEMÁNTICO CATEGORIAL
}

\author{
F. JAVIER MORENO ${ }^{1}$ Y SARA CAÑAMÓN ${ }^{2}$ \\ 1 Universidad Nacional de Educación a Distancia \\ ${ }^{2}$ Universidad de Alcalá, Madrid.
}

(Aceptado en junio de 2005)

\begin{abstract}
El estudio de las disociaciones categoriales de los dominios de los seres vivos y de los seres no vivos o cosas fabricadas por el hombre es de gran interés para investigar la organización, el funcionamiento y la posible localización cerebral de la memoria semántica. La mejor estrategia para estudiar este fenómeno es utilizar baterías semánticas que constan de varias pruebas y utilizan los mismos items presentados bajo diferentes modalidades. En este trabajo se presenta un nuevo instrumento: la batería Nombela, que pretende satisfacer una demanda entre las pruebas neuropsicológicas españolas, pues con excepción de la batería EMSDA (Peraita et al., 2000) no existe ningún instrumento en español para evaluar el deterioro semántico. Además, presenta mejoras importantes respecto a instrumentos anteriores: emplea, al estudiar 14 categorías semánticas, categorías de interés teórico, y objetos que varían en su grado de manipulabilided. Finalmente, controla variables importantes en el estudio del deterioro semántico.
\end{abstract}

Palabras clave: Deterioro de categorías específicas, baterfas semánticas, patología cerebral, enfermedad de Alzheimer.

\section{Presentation and preliminary results of Semantic Battery Nombela (I). A new instrument to evaluate semantic categorial impairment}

The study of categorical dissociations between the domains of living thing (LT) and nonliving thing (NLT) is highly relevant to the investigation of the organization and functioning of semantic memory, as well as its possible brain localization. Semantic batteries are the best approach for studying these matters. These instruments use different semantic tasks, but elaborated with the same items, in order to get convergent evidence from them. We present a new instrument: the Semantic Battery Nombela, which is intended to enrich Spanish neuropsychological assessment since, to our knowledge, there are not many Spanish instruments to evaluate specifically semantic memory impairments, with the exception of the semantic battery, EMSDA (Peraita et al., 2000). Furthermore, our battery tries to improve other similar instruments by studying 14 semantic categories, new theoretically interesting categories, and objects with a different range of manipulability. Finally, the items of the battery were controlled in some important variables.

Keywords: Category-specific impairment, categorial dissociations, semantic batteries, brain pathology, Alzheimer disease.

Correspondencia: F. Javier Moreno, Universidad Nacional de Educación a Distancia (UNED), Facultad de Psicología, Departamento de Psicología Básica I, Juan del Rosal,10, 28040 Madrid. Correo-e: fjmoreno@psi.uned.es

Agradecimientos: Deseamos mostrar nuestro agradecimiento al Dr. Miguel Goñi, del Hospital Divino Vallés (Burgos) y a la Profesora Herminia Peraita, de la UNED.

\section{INTRODUCCIÓN}

Hace sesenta años, Nielsen (1943) describió dos pacientes con daño cerebral que presentaban un extraño patrón de deterioro. Uno de ellos tenía dificultades con los objetos animados (como los animales) aunque no con los objetos inanimados 
(como las herramientas). El segundo mostraba la disociación opuesta: el deterioro específico de los objetos inanimados, pero la conservación de los animados. La lesión del primero afectó principalmente al lóbulo occipital izquierdo, mientras que el segundo presentó daños en el derecho. Por este motivo, Nielsen pensó que el sistema de procesamiento de los items de los dominios animado e inanimado tenía una localización cerebral precisa, situándose en el lóbulo occipital. Pese a que recientes investigaciones han demostrado que la propuesta de Nielsen no es correcta, sus ideas «localizacionistas» serán retomadas por modelos semánticos más actuales (Caramazza y Shelton, 1998). Cuatro décadas después, Warrington y Shallice (1984) estudiaron varios pacientes con deterioro en la denominación e identificación de los seres vivos (SV), respecto a los seres no vivos o cosas fabricadas por el hombre (SNV). Desde entonces, los estudios sobre este asunto han ido incrementándose progresivamente (ver Capitani, Laiacona, Mahon y Caramazza, 2003; Martin y Caramazza, 2003). Estos hallazgos son interesantes debido a sus implicaciones para el estudio de la organización de la memoria semántica (MS) y de su posible localización cerebral.

Una de las primeras explicaciones para este fenómeno sugirió que no era más que un artefacto metodológico, al observarse que los items del dominio de los SV - cuyo deterioro es el más habitualson más difíciles de procesar que los del dominio de los SNV. Varios autores verificaron que los items de los SV eran más complejos visualmente, menos familiares y menos típicos que los items de los SNV, por lo que no había razones para pensar en un verdadero deterioro categorial (Funnell y Sheridan, 1992; Gaffan y Heywood, 1993; Stewart, Parkin y Hunking, 1992). Si bien existen variables que afectan al procesamiento de los items y que, en general, perjudican a los SV, también es cierto que han seguido hallándose ese tipo de deterioros, incluso en estudios metodológicamente correctos (ver Capitani et al., 2003). Otro hallazgo que desautorizó la explicación metodológica fue el descubrimiento de deterioros de los SNV, cuyo procesamiento es teóricamente más sencillo (Hillis y Caramazza, 1991).

Es probable que algunos estudios que informaron de efectos categoriales - sobre todo los más antiguos- no realizaran un buen control metodológico de los items, más teniendo en cuenta el desconocimiento de la influencia de variables como la frecuencia léxica, la familiaridad o la complejidad visual, entre otras. Si bien es cierto que todas las disociaciones categoriales - también llamadas deterioros categoriales - no son atribuibles a problemas metodológicos, no lo es menos que la falta de control de tales variables puede producir resultados espurios. Incluso hoy en día siguen apareciendo trabajos que no han controlado muchas de estas variables (Brousseau y Buchanan, 2005; Bunn, Tyler y Moss, 1998; Gonnerman, Andersen, Devlin, Kempler y Seidenberg, 1997; Montanes, Goldbum y Boller, 1995, 1996). Tal y como propusieron Barbarotto, Capitani y Laiacona (2001), sería bueno conocer la validez de muchos estudios que no controlaron este tipo de variables.

Una de las cuestiones que comienza a plantearse en los estudios sobre deterioro categorial está muy relacionada con lo expuesto. Nos referimos a la posibilidad de que exista una verdadera ventaja en el procesamiento de los items del dominio de los SNV. Por ejemplo, en un estudio bien controlado, Zannino, Perri, Carlesimo, Pasqualetti y Caltagirone (2002) verificaron un mayor deterioro de alguno de sus enfermos de Alzheimer en el dominio de los SV; sin embargo, los participantes controles también mostraron una tendencia en esa dirección. Zanino et al. 
(2002) reconocieron que la buena actuación de los participantes sanos, cercana al efecto techo $(\mathrm{SV}=98 \%, \mathrm{SNV}=98,3$ $\%)$, no les permitió indagar sobre este importante hallazgo. Algo parecido fue verificado por Moreno y Peraita (2005a), que también hallaron una ventaja en el procesamiento de los SNV frente a los $\mathrm{SV}$, tanto en un grupo de pacientes de Alzheimer como en los controles sanos. No obstante, es interesante indicar que, a diferencia del estudio de Zanino et al. (2002), los participantes sanos de Moreno y Peraita (2005a) no alcanzaron el efecto techo ( $\mathrm{SV}=89 \%, \mathrm{SNV}=93 \%$ ). Esto les llevó a concluir que los individuos sanos también pueden mostrar un mejor rendimiento con los SNV, y que este hallazgo podría ser la tendencia normal. Si esto es así, puede que el hecho de no hallar más resultados en esa dirección esté relacionado con el nivel de dificultad de las tareas, ya que si los participantes sanos alcanzan el efecto techo es difícil detectar tal tendencia (Laws, Gale, Leeson y Crawford, 2005). El problema del efecto techo se relaciona con el número de categorías normalmente estudiadas y con la clase de items utilizados en las tareas de evaluación. Es recomendable que los instrumentos que evalúen el deterioro semántico tengan en cuenta esos factores.

Las categorías más utilizadas en los estudios neuropsicológicos son las de animales, frutas y verduras, del dominio de los SV, y las de herramientas, muebles y vehículos, del de los SNV. Tal y como se ha demostrado (Azuma et al., 1997; Diaz, Sailor, Cheung y Kuslansky, 2004), existen importantes diferencias entre las categorías, tanto en el número de los elementos que las forman como en su comportamiento frente al daño cerebral (Diaz et al., 2004). También se sabe que la sensibilidad y fiabilidad de los resultados aumenta cuando se utilizan varias categorías para evaluar el conocimiento semántico (Monsch, Bondi, Butters, Salmon, Katzman. y Thal, 1992). Las razones expuestas sugieren que una buena exploración semántica debería utilizar más categorías que las habitualmente estudiadas.

Otra cuestión polémica tiene que ver con la existencia de categorías "problemáticas» o "atípicas» (Gale, Laws y Foley, en prensa). Tal y como se comentó, la mayoría de los estudios han utilizado las categorías de animales, frutas y verduras, del dominio de los SV, y las de herramientas, muebles y vehículos, del de los SNV (ver Capitani et al., 2003). Sin embargo, existen categorías que están envueltas en cierta polémica teórica: los instrumentos musicales y las partes del cuerpo. En teoría, los instrumentos musicales pertenecen al dominio de los SNV, mientras que las partes del cuerpo pertenecen al de los SV. Sin embargo, varios estudios han verificado que, en términos de deterioro, ambas categorías se encuadran dentro del dominio opuesto. El patrón de conservación y/o deterioro de las partes del cuerpo se asemeja al de los SNV y el de los instrumentos musicales al de los SV (para una revisión, ver Capitani et al., 2003). Esto ha llevado a que algunos autores hayan decidido $a$ priori no estudiar ambas categorías (Garrard, Patterson, Watson y Hodges, 1998; Laws, 1999). Incluso, hay quienes proponen explícitamente su exclusión de las investigaciones sobre deterioro categorial (Coppens y Frisinger, 2005). En nuestra opinión, el hecho de que existan temas polémicos no es motivo para que sean apartados de la investigación, sino que deben estudiarse para intentar su esclarecimiento.

Otra cuestión importante es que la dicotomía vivo/no vivo quizá no sea suficiente para captar toda la complejidad del fenómeno de las disociaciones categoriales. Algunos autores piensan que la disociación vivo/no vivo debería sustituirse 
por un nuevo término: animado/inanimado. Caramazza y sus colaboradores proponen que nuestro sistema cognitivo está especializado en el procesamiento (reconocimiento y clasificación) de los ejemplares pertenecientes a ciertos dominios, por su importancia para la supervivencia de la especie: animales, partes del cuerpo, plantas, congéneres y herramientas. Según estas ideas, es necesario reconocer rápidamente si un fruto es apto para el consumo, o si un animal es un depredador o un alimento potencial (Caramazza y Shelton, 1998; Shelton, Fouch y Caramazza, 1998). Este enfoque enfatiza el papel de las presiones medioambientales que a lo largo del tiempo han generado mecanismos de procesamiento especializados. De este modo, si el daño cerebral afecta al subsistema que procesa determinados items (p.ej., los animales), se producirá su deterioro. Para poder investigar este asunto debe contarse con items de los dominios mencionados.

La mayoría de los estudios que han evaluado el conocimiento semántico o conceptual han utilizado tareas que se supone valoran este conocimiento. Muchas de estas tareas se presentan juntas, dentro de las llamadas baterías de evaluación semántica, cuya principal característica es utilizar los mismos items presentados bajo diferentes modalidades. Se pretende evaluar tanto la entrada (input) como la salida (output) de información del sistema semántico (ver p.ej., Hodges, Graham y Patterson, 1995; Laiacona, Barbarotto, Trivelli y Capitani, 1993; Peraita, González-Labra, Sánchez y Galeote, 2000). Estas baterías permiten explorar el sistema semántico de forma precisa, al obtener evidencia convergente de distintas tareas (Bright, Moss y Tyler, en prensa).

Tal y como se ha expuesto con anterioridad, un instrumento que pretenda evaluar con garantía la MS debe cumplir una serie de requisitos. El primero es contro- lar variables como la frecuencia léxica, la complejidad visual o la tipicidad, entre otras, que influyen en el rendimiento de los participantes (Lambon Ralph, Howard, Nightingale y Ellis, 1998). De esta forma, nos aseguramos de que los efectos se deben al deterioro causado por la enfermedad y no a sesgos provocados por los items. También es importante examinar un buen número de categorías de ambos dominios (SV y SNV), ya que esto nos proporcionará una visión más detallada y completa del conocimiento semántico, sobre todo teniendo en cuenta la variabilidad existente entre las categorías (Azuma et al., 1997; Díaz et al., 2004). En estrecha relación con lo anterior, está el problema del efecto techo: es interesante estudiar categorías de ambos dominios que dificulten que los participantes sanos alcancen niveles cercanos al $100 \%$ de exactitud. Otro de los requisitos de un buen instrumento de evaluación es la incorporación de categorías de interés teórico, como las de partes del cuerpo e instrumentos musicales. Finalmente, deben estudiarse nuevas categorías, teóricamente interesantes, como los árboles y las flores (Caramazza y Shelton, 1998, McRae y Cree, 2002), los insectos (McRae y Cree, 2002), y SNV que varien en su grado de manipulabilidad (Magnié, Besson, Poncet y Dolisi, 2003; Warrington y McCarthy, 1987).

En este trabajo se presenta un nuevo instrumento de evaluación neuropsicológica que cumple esos requisitos. La batería Nombela evalúa el deterioro semántico categorial en pacientes con daño cerebral y explora el fenómeno de los efectos categoriales en la población sana. Este instrumento pretende satisfacer una demanda entre las pruebas neuropsicológicas españolas, pues con excepción de la batería EMSDA (Peraita et al., 2000) no existe ningún instrumento en español para evaluar el conocimiento léxicosemántico. Pese al incuestionable valor 
de la batería EMSDA, herramienta pionera en España en este campo, sus items sólo fueron controlados en la variable tipicidad (según Soto, Sebastián, García y del Amo, 1994). De igual modo, sólo explora seis categorías semánticas y no incluye items de partes del cuerpo ni de instrumentos musicales.

Una de las principales innovaciones de la batería Nombela es utilizar items controlados en variables importantes. Concretamente, los items que componen esta batería presentan normas de siete variables intrínsecas. A diferencia de otras baterías, la mayoría de estas normas se obtuvieron de ancianos sanos (ver Moreno y Peraita, 2005b). Que nosotros sepamos, esta es la primera batería semántica que presenta tal cantidad de índices obtenidos de una misma población. Asimismo, en la prueba de denominación de fotografías de la batería Nombela, los participantes sanos no alcanzan el efecto techo (Moreno y Peraita, 2005a).

En este trabajo se presentan todas las pruebas que componen la batería y los resultados provisionales de cinco de ellas, tras evaluar a un grupo de ancianos sanos y a otro de pacientes de Alzheimer: clasificación de fotografías (nivel de dominio), decisión de objetos, denominación de fotografías, emparejamiento palabra-fotografía y fluidez semántica.

\section{MÉTODO}

\section{Participantes}

Participaron 31 personas divididas en dos grupos: un grupo experimental de 15 pacientes de Alzheimer ( 8 mujeres y 7 hombres; edad media $=74,6$ años; $\mathrm{D} T$ $=6,8 ;$ media de años de educación $=9$; DT $=5$; puntuación media en el Mini Mental State Examination (MMSE) = 21,4; DT $=3$ ), y un grupo de control de 16 ancianos sanos ( 8 mujeres y 8 hom- bres; edad media $=71,1$ años; DT $=7,7$; media de años de educación $=8,8 ; \mathrm{DT}=$ 3,9; puntuación media en el MMSE = 29; DT $=1,9$ ).

Los pacientes fueron diagnosticados de enfermedad de Alzheimer (EA) probable según los criterios NINCDS-ADRDA (National Institute of Neurological and Communication Disorders and StrokeAlzheimers-Disease-and-Related-Disorders-Association) por neurólogos de varios hospitales españoles. A todos se les aplicaron las pruebas complementarias recomendadas por el grupo de Neurología de la Conducta y Demencia de la Sociedad Española de Neurología (SEN), para excluir otras enfermedades. El grupo experimental no difirio significativamente del grupo de control en edad o nivel educativo $(p>0,05)$. Sin embargo, tal y como era previsible, las puntuaciones en el MMSE de Folstein, Folstein y McHugh (1975), tras aplicar las correcciones para edad y nivel educativo para población española (Blesa et al., 2001), fueron significativamente inferiores en el grupo de pacientes $(p<0,001)$.

Ningún participante mostraba trastornos psiquiátricos, enfermedad médica, o cualquier otra enfermedad neurológica -excluyendo la propia EA - que pudiera afectar la actuación cognitiva. Los 31 participantes eran hablantes-oyentes de castellano.

\section{La batería Nombela}

La batería Nombela es un instrumento de evaluación neuropsicológica formado por diversas pruebas que evalúan el deterioro semántico categorial, y que han sido validadas por un amplio número de estudios neuropsicológicos (Capitani et al., 2003; Daum, Riesch, Sartori y Birbaumer, 1996; Hodges et al., 1995; Laiacona et al., 1993; Peraita et al., 2000). Incluye pruebas visuales y verbales que exploran 
tanto el estado de la MS como otros niveles de procesamiento - perceptuales, léxicos y fonológicos. Está compuesta por las siguientes tareas:

1. Dos tareas de denominación: una de denominación de fotografías y otra de denominación tras descripción, que evalúan la capacidad de denominación de los individuos mediante dos tipos de input: pictóricos, la primera tarea y verbales, la segunda.

2. Cuatro tareas que evalúan el procesamiento semántico en condiciones que no requieren la recuperación del nombre del ítem: tareas de clasificación, emparejamiento y escalamiento por tamaño, con items pictóricos, y tarea de analogías semánticas, con items verbales.

3. Una tarea que evalúa el procesamiento léxico-semántico mediante la producción de ejemplares de categorías semánticas: tarea de fluidez semántica categorial.

4. Tres tareas que evalúan el procesamiento perceptual, léxico y fonológico de los participantes. Para la detección de dificultades léxicas y viso-perceptuales se utilizan las tareas de decisión de palabras y de decisión de objetos, respectivamente. Las dificultades fonológicas se evalúan de dos maneras: a) haciendo que los participantes lean en voz alta los items de la tarea de decisión léxica y, b) haciendo que repitan los nombres de los items en la tarea de escalamiento por tamaño.
Los items y categorías que se utilizan en las tareas de la batería se han descrito en trabajos anteriores (Moreno y Peraita, 2003; 2005b; en prensa; Moreno, Peraita, Cañamón y Silva, 2004). La batería emplea 112 items que pertenecen a 14 categorías semánticas, siete del dominio de los SV: animales, árboles, flores, frutas, insectos, partes del cuerpo y verduras; y siete del dominio de los SNV: edificios, herramientas, instrumentos musicales, muebles, prendas de vestir, utensilios de cocina y vehículos. Cada categoría está compuesta por ocho items de los que se han obtenido índices de las siguientes variables intrínsecas: acuerdo en el nombre, complejidad visual, edad de adquisición del nombre del objeto, familiaridad, frecuencia léxica, manipulabilidad del objeto y tipicidad (en Moreno y Peraita, 2005b, se detalla el procedimiento seguido para obtener estos índices). En la Tabla 1 se detallan las tareas, así como el orden en el que se presentaron a los participantes para minimizar los efectos de priming. La evaluación se realizó de forma individual.

\section{Descripción de las tareas}

1. Tarea de fluidez semántica categorial. En esta tarea se pidió a cada participante que, durante un periodo de un minuto, generase tantos ejemplares como pudiera de cada una de las 14 categorías. El orden de las categorías se organizó para que no se presentaran de forma consecutiva dos del mismo dominio. Del

Tabla 1. Tareas experimentales y orden en el que se presentaron a los participantes.

\begin{tabular}{ll}
\hline \multicolumn{1}{c}{ PRIMERA SESIÓN } & \multicolumn{1}{c}{ SEGUNDA SESIÓN } \\
\hline 1. Fluidez semántica categorial. & 6. Denominación tras descripción. \\
2. Denominación de fotografías. & 7. Clasificación (Categoría). \\
3. Emparejamiento palabra-fotografía. & 8. Analogías semánticas. \\
4. Decisión de objetos. & 9. Decisión de palabras. \\
5. Clasificación (Dominio). & 10. Clasificación (Atributo). \\
& 11. Escalamiento por tamaño. \\
\hline
\end{tabular}


total de los items generados se descontaron las repeticiones e intrusiones; una intrusión se definió como cualquier ítem que no perteneciera a la categoría en cuestión (incluir «rosal» en la categoría de los árboles). Además, solamente se aceptaron los items referidos a una subcategoría (p.ej., pájaros) cuando el participante no generó ningún ejemplar específico de esa subcategoría (p.ej., gorriones o águilas) (Capitani, Laiacona y Barbarotto, 1999). Las respuestas se grababan para su posterior análisis.

2. Tarea de denominación de fotografías. Mediante la pantalla de ordenador se presentaban fotografías a color de los 112 estímulos experimentales. Los items se presentaban de forma aleatoria y de uno en uno, permanecían en la pantalla hasta que el participante generaba una respuesta y eran seguidos por un intervalo entre estímulos de tres segundos. Cuando un participante no generaba una respuesta, se le animaba a que dijera el nombre de la categoría del ítem mediante la frase: ¿cómo se llama el grupo de cosas al que pertenece ese objeto o cosa? Si no respondía, se dejaban transcurrir 10 segundos antes de pasar al siguiente ítem. Las respuestas se anotaban en el cuadernillo de respuesta para su posterior análisis.

3. Tarea de denominación tras descripción. En esta tarea el participante tenía que decir el nombre del ítem tras proporcionarle una descripción verbal del mismo, del tipo: ¿cómo se llama un animal doméstico que caza ratones? Había 56 definiciones, 28 de cada dominio. Las definiciones se presentaban de forma oral, sin que aparecieran seguidas dos del mismo dominio. Las respuestas se anotaban en el cuadernillo para su posterior análisis.

4. Tarea de emparejamiento palabra oída-fotografía. Mediante la pantalla de ordenador se presentaban a cada participante los ocho items de cada categoría conjuntamente. El participante tenía que señalar el estímulo target nombrado por el experimentador, la tarea continuaba hasta que el participante señalaba todos los items de esa categoría, y así sucesivamente con el total de las categorías. El orden de las categorías se organizó para que no se presentaran de forma consecutiva dos del mismo dominio; además, se aleatorizó el orden en el que el experimentador nombraba cada ítem. Las respuestas se anotaban en el cuadernillo para su posterior análisis.

5. Tarea de clasificación de fotografías. En esta tarea había 84 items, 42 de cada dominio. Los items eran fotografías a color y se presentaban en tarjetas de $12,5 \times 9,5 \mathrm{~cm}$. Tal y como muestra la Tabla 2, la tarea tuvo tres niveles de dificultad (Hodges et al., 1995); cada nivel se realizaba sucesivamente del más sencillo al más difícil, sin límite temporal. En el primer nivel (nivel de dominio o supraordenado), el participante tenía que clasificar los items en dos grupos: 1) dominio de los SV o natural y, 2) dominio de los SNV o artificial. En el siguiente nivel de dificultad (nivel de categoría o básico), tenía que clasificarlos según su categoría de pertenencia: animales, árboles, flores, y así sucesivamente. Finalmente, en el tercer nivel (nivel de atributo o subordinado), el participante tenía que clasificar los items de cada categorfa en dos grupos. Por ejemplo, para la categoría de animales en: 1) domésticos y, 2) salvajes. Las respuestas se anotaban en el cuadernillo para su posterior análisis.

6. Tarea de analogías semánticas. En esta tarea los participantes tenían que realizar un razonamiento analógico, es decir, establecer nuevas relaciones semánticas a partir de una analogía determinada que servía como ejemplo. Los participantes tenían que detectar la relación semántica existente entre items que guardan entre sí una relación del tipo: $A$ es a $B$ como $C$ es a D. Los dos primeros términos (A y B) 
Tabla 2. Estructura de la tarea de clasificación de fotografías.

\begin{tabular}{|c|c|}
\hline \multicolumn{2}{|c|}{ NIVEL DE DOMINIO O SUPRAORDENADO } \\
\hline Vivo o Natural & No vivo o Artificial \\
\hline \multicolumn{2}{|c|}{ NIVEL DE CATEGORÍA O BÁSICO } \\
\hline 1. Animales & 8. Edificios \\
\hline 2. Árboles & 9. Herramientas \\
\hline 3. Flores & 10. Instrumentos musicales \\
\hline 4. Frutas & 11. Muebles \\
\hline 5. Insectos & 12. Prendas de vestir \\
\hline 6. Partes del cuerpo & 13. Utensilios de cocina \\
\hline 7. Verduras & 14. Vehículos \\
\hline
\end{tabular}

NIVEL DE ATRIBUTO O SUBORDINADO

Pertenecientes al dominio de los SV

1. Animales: domésticos / salvajes.

2. Árboles: frutos comestibles / frutos no comestibles.

3. Flores: muy olorosas / poco olorosas.

4. Frutas: crecen en el suelo / crecen en los árboles.

5. Insectos: venenosos / no venenosos.

6. Partes del cuerpo: son articulaciones / no son articulaciones.

7. Verduras: de hojas o cuerpo duro / de hojas o cuerpo tierno.
Pertenecientes al dominio de los SNV

1. Edificios: monumentos / no monumentos.

2. Herramientas: sirven para cortar / no sirven para cortar.

3. Instrumentos musicales: viento / cuerda.

4. Muebles: típicos del dormitorio / típicos del salón-comedor.

5. P. vestir: parte superior del cuerpo / p. inferior del cuerpo.

6. U. cocina: se utilizan para comer / no se utilizan para comer.

7. Vehículos: transporte público / transporte privado. mantienen una relación explícita en la analogía, y el participante debía descubrir el término desconocido (D) que mantiene una relación con $\mathrm{C}$, análoga a la primera relación. En esta tarea había 28 items, 14 de cada dominio. Existían tres distractores, dos de ellos pertenecientes al mismo dominio y distinta categoría que el target, y uno de distinto dominio (ver Figura 1). Los items se presentaban mediante la pantalla de ordenador y las respuestas se

\begin{tabular}{|c|c|}
\multicolumn{2}{|c|}{$\begin{array}{c}\text { MARGARITA } \\
\text { ROSA }\end{array}$} \\
\hline AMAPOLA & CLAVEL \\
\hline PIERNA & LÁMPÁN \\
\hline
\end{tabular}

Figura 1. Ejemplo de un ítem de la tarea de analogías pertenecientes a la categoría flores. anotaban en el cuadernillo para su posterior análisis.

7. Tarea de escalamiento por tamaño. En esta tarea había 53 items 25 del dominio de los SV y 28 del de los SNV. El experimentador colocaba delante del participante todos los items de una misma categoría (fotografías a color en tarjetas de $12,5 \times 9,5 \mathrm{~cm}$.). Posteriormente, señalaba cada ítem, lo nombraba y pedía al participante que repitiera el nombre del mismo. Después de esto, le solicitaba que eligiera la fotografía que mostrara el ítem de mayor tamaño o volumen, teniendo en cuenta que su juicio debía basarse en el tamaño o volumen del ítem en la vida real, y no en el representado en la fotografía. Después de elegir una fotografía, ésta se retiraba de la mesa y el participante tenía que elegir, de entre las restantes, el ítem de mayor tamaño, y así sucesivamente hasta seleccionar todos los items. Este procedimiento se repetía con todas las 
categorías. Las respuestas se anotaban en el cuadernillo para su posterior análisis.

8. Tarea de decisión de objetos. Mediante la pantalla de ordenador se presentaban de forma aleatoria 64 items (32 reales y 32 quiméricos). Los items quiméricos son dibujos lineales en blanco y negro (Barbarotto, Laiacona, Macchi y Capitani, 2002). Se utilizaron cuatro items pertenecientes a cada una de las categorías semánticas estudiadas por Barbarotto et al. (2002): animales, frutas, partes del cuerpo y verduras, del dominio de los SV, y herramientas, instrumentos musicales, muebles y vehículos, del dominio de los SNV. Los 32 items reales se tomaron del corpus de Snodgrass y Vanderwart (1980), se seleccionaron los cuatro items de mayor acuerdo en la denominación de su categoría según las normas españolas de Sanfeliú y Fernández (1996). La tarea de los participantes consistía en decir si el ítem presentado era real o quimérico. Los items permanecían en la pantalla hasta que se daba una respuesta y eran seguidos de un intervalo entre estímulos de dos segundos. En caso de que el participante no diera ninguna respuesta se dejaban transcurrir 10 segundos antes de pasar al siguiente ítem. Las respuestas se anotaban en el cuadernillo para su posterior análisis.

9. Tarea de decisión de palabras. Mediante la pantalla de ordenador se presentaban 42 palabras y 42 seudo-palabras, de forma aleatoria. Las palabras correspondían a los nombres de 42 estímulos experimentales, 21 de cada dominio. Las 42 seudo-palabras se construyeron alterando el orden de los fonemas de los nombres de los items, de forma que se ajustaran a las reglas fonológicas del español. La tarea de los participantes consistía en leer en voz alta el nombre del ítem, y decir si era o no una palabra real. El hecho de que los participantes leyeran en voz alta el nombre de cada ítem sirvió para evaluar su procesamiento fonologico. Las respuestas se anotaban en el cuadernillo para su posterior análisis.

\section{RESULTADOS}

Para todas las tareas, con excepción de las de fluidez semántica y decisión de objetos, se realiź un análisis de regresión múltiple para cada uno de los dos grupos de participantes (hay que indicar que en la tarea de clasificación, los ancianos sanos no cometieron errores, por lo que sólo se analizaron los datos de los pacientes) (Perri et al., 2003; Silveri, Cappa, Mariotti y Puopolo, 2002; Zannino et al., 2002). La variable dependiente fue la proporción grupal de aciertos en las tareas. Las variables independientes -o predictoras - de la denominación fueron las siete variables intrínsecas: acuerdo en el nombre, complejidad visual, edad de adquisición del nombre, familiaridad, manipulabilidad del objeto, frecuencia léxica y tipicidad; la variable Dominio se codificó como variable

Tabla 3. Puntuaciones medias obtenidas por los ancianos sanos (AS) y los pacientes de Alzheimer (PA) en las cinco tareas analizadas.

\begin{tabular}{|c|c|c|c|c|c|c|c|c|c|c|}
\hline & \multicolumn{2}{|c|}{ Fluidez } & \multicolumn{2}{|c|}{$\begin{array}{l}\text { Denominación } \\
\text { fotografías }\end{array}$} & \multicolumn{2}{|c|}{$\begin{array}{l}\text { Emparejamiento } \\
\text { palabra-fotografía }\end{array}$} & \multicolumn{2}{|c|}{$\begin{array}{l}\text { Decisión } \\
\text { de objetos }\end{array}$} & \multicolumn{2}{|c|}{$\begin{array}{c}\text { Clasificación } \\
\text { dominio }\end{array}$} \\
\hline & SV & SNV & SV & SNV & SV & SNV & SV & SNV & SV & SNV \\
\hline AS & $11,1(0,5)$ & $10,5(0,5)$ & $0,9(0,01)$ & $0,92(0,01)$ & $0,98(0,01)$ & $0,99(0,01)$ & $0,94(0,01)$ & $0,95(0,01)$ & $1(0)$ & $1(0)$ \\
\hline PA & $5,1(0,5)$ & $5,5(0,5)$ & $0,73(0,02)$ & $0,76(0,02)$ & $0,88(0,02)$ & $0,88(0,02)$ & $0,88(0,02)$ & $0,88(0,02)$ & $0,88(0,01)$ & $0,99(0,01)$ \\
\hline
\end{tabular}

Nota: " Media (y desviación típica) de los elementos producidos. ${ }^{b}$ Proporción media (y desviación típica) de respuestas correctas. 
dummy ( $\mathrm{SV}=1, \mathrm{SNV}=0)$. La Tabla 3 muestra las puntuaciones de los dos grupos de participantes en ambos dominios en las cinco tareas.

\section{Análisis de varianza}

Tarea de fluidez semántica categorial. El ANOVA mostró un efecto principal significativo del Grupo $(F(1,29)=61,71$; $p<0,001)$. La interacción Grupo x Dominio resultó significativa $(F(1,29)=5,08$; $p<0,05$ ). El análisis post hoc (Bonferroni) mostró que la interacción fue debida al mayor deterioro de los pacientes en el dominio de los $S V(p<0,001)$.

Tarea de decisión de objetos. El ANOVA mostró un efecto principal significativo del Grupo $(F(1,29)=9,39 ; p<0,005)$, con un mejor rendimiento de los ancianos sanos. No hubo efectos del Dominio ni interacciones significativas.

\section{Análisis de regresión múltiple}

Tarea de denominación de fotografías. En el grupo de pacientes, las variables acuerdo en el nombre del objeto $(t=9,6 ; p$ $<0,001)$, edad de adquisición del nombre $(t=-2,97 ; p<0,005)$ y manipulabilidad del objeto $(t=2,06 ; p<0,05)$ predijeron significativamente la denominación de los items. El porcentaje de varianza explicada por el modelo fue del $78 \%\left(R^{2}=0,78\right)$. En el grupo de ancianos la única variable que predijo la denominación fue el acuerdo en el nombre del objeto $(t=13,13 ; p<0,001)$. El porcentaje de varianza explicada por el modelo fue del $80 \%\left(R^{2}=0,8\right)$. El dominio de los items (SV o SNV) no contribuyó a la predicción de la denominación en ninguno de los dos grupos.

Tarea de emparejamiento palabra oídafotografía. En el grupo de pacientes, las variables acuerdo en el nombre del objeto $(t=5 ; p<0,001)$ y manipulabilidad del objeto $(t=2,86 ; p<0,006)$ predijeron significativamente la denominación de los items. El porcentaje de varianza explicada por el modelo fue del $50 \%\left(R^{2}=0,5\right)$. En el grupo de ancianos, las variables tipicidad $(t=-2,3 ; p<0,05)$ y acuerdo en el nombre del objeto $(t=3,9 ; p<0,001)$ predijeron la denominación. El porcentaje de varianza explicada por el modelo fue del $30 \%\left(R^{2}=0,3\right)$. El dominio de los items (SV o SNV) no contribuyó a la predicción de la denominación en ninguno de los dos grupos.

Tarea de clasificación de fotografías (nivel de dominio). Las variables frecuencia léxica de los items $(t=2,88 ; p<0,006)$ y manipulabilidad del objeto $(t=-3,68 ; p$ $<0,001)$ predijeron significativamente la denominación de los items. El valor negativo del coeficiente beta $(\beta)$ reveló que los pacientes clasificaron mejor los items del dominio de los SNV.

\section{DISCUSIÓN}

El propósito de este trabajo fue presentar la batería Nombela, un nuevo instrumento que pretende satisfacer una demanda dentro del panorama neuropsicológico español, donde no se cuenta con muchas pruebas de esta índole, con algunas notables excepciones (Peraita et al., 2000). El objetivo de la batería Nombela es investigar el deterioro específico de categoría o disociación categorial de los dominios de SV y SNV. Este fenómeno tiene una gran importancia teórica, ya que sugiere que el conocimiento semántico posee cierta estructura interna y, además, indica que sus componentes pueden depender de diferentes sustratos neuronales (Thompson-Schill, 2003).

En general, los resultados tras evaluar un grupo de ancianos sanos y otro de pacientes de Alzheimer son consistentes con anteriores estudios neuropsicológicos. Como se observó, el rendimiento del 
grupo de pacientes fue inferior en todas las tareas. Los pacientes mostraron deterioros significativos en una tarea puramente verbal -fluidez semántica-, en tareas viso-verbales, -clasificación por dominio, denominación de fotografías y emparejamiento palabra-fotografía-, y en tareas puramente visuales que requieren decisiones sobre si un ítem es quimérico o real -decisión de objetos-. Sin embargo, solamente en dos de estas tareas -fluidez y clasificación por dominio-se verificó la presencia de deterioro categorial, observándose un peor rendimiento con los $\mathrm{SV}$.

Algunos autores han sugerido una explicación para el deterioro semántico en la EA, basándose en el tipo de procesamiento requerido por la tarea. Se afirma que el hecho de hallar o no deterioro semántico podría estar relacionado con el nivel de procesamiento o «esfuerzo cognitivo" requerido por la tarea (Nebes, 1989; Nebes, Martin y Horn, 1984; Ober y Shenaut, 1995). Los pacientes tendrían una buena actuación en las tareas que requieren un escaso nivel de procesamiento (priming), en cambio, en aquellas que requieren un nivel más alto (fluidez semántica) mostrarían deterioro semántico. Según esta idea, algunos autores han propuesto que el hecho de hallar deterioro categorial en la EA está relacionado con la dificultad de la tarea, de forma que solamente se hallarán deterioros de los $\mathrm{SV}$ en las tareas más difíciles, esto es, en las que requieren un nivel más alto de procesamiento cognitivo (Daum et al., 1996). Ya que se ha establecido que los items de los SNV se procesan "mejor» que los items de los SV (Sheridan y Humphreys, 1993), se sugiere una interacción entre la dificultad de las tareas y la "cantidad" de procesamiento requerido por los items. No obstante, esta explicación resulta menos apropiada cuando se produce el deterioro de los SNV, patrón que, aunque menos habitual, tam- bién se ha hallado (ver Capitani et al., 2003).

La explicación anterior no encaja con nuestros datos; si nos atenemos a los procesos que ponen en juego las tareas, el orden teórico de las pruebas de mayor a menor nivel dificultad sería: 1) fluidez semántica, 2) denominación, 3) decisión de objetos, 4) emparejamiento palabrafotografía y 5) clasificación por dominio. Los datos empíricos demostraron que en la tarea de fluidez semántica los ancianos sanos generaron una media de 10,8 items y los pacientes una media de 5,3 items, es decir, los pacientes mostraron una pérdida del $51 \%$ frente a los ancianos sanos. Este porcentaje de deterioro fue del $17,6 \%, 10,2 \%, 7,4 \%$ y $6 \%$ en las tareas de denominación de fotografías, emparejamiento palabra-fotografía, decisión de objetos y clasificación por dominio, respectivamente. Es decir, los pacientes de Alzheimer mostraron efectos categoriales tanto en una tarea «difícil» -fluidez semántica- como en una «fácil»-clasificación-, por lo que nuestros hallazgos no sugieren una interacción entre el nivel de dificultad de la tarea y la presencia -o ausencia- de deterioro categorial, en línea con lo sugerido por otros autores (Daum et al., 1996; Rich, Park, Dopkins y Brandt, 2002).

Otro hallazgo interesante fue que el grupo de pacientes tuviera un peor rendimiento al clasificar los items de los SV, resultado que difiere de lo hallado en otros trabajos (Montanes et al., 1996). Se ha sugerido que existe un mayor parecido intra-categorial de los items de los SV (Gaffan y Heywood, 1993). Esta mayor similitud estructural debería favorecerles en la tarea de clasificación a nivel supraordenado, cuyo desempeño se basa en las características más generales o compartidas de los items. Por este motivo, los items de los SV deberían clasificarse mejor que los items de los SNV, que comparten menos características. Además, el 
hecho de utilizar items a color debería haber favorecido - aún más-a los items de los SV: si es más fácil clasificar items de este dominio, la diferencia entre ambos dominios tendría que incrementarse cuando reciben una "ayuda» extra (el color), algo que no sucedió en nuestro estudio. No obstante, recordemos que los ancianos sanos no cometieron errores en esta tarea, lo que nos impidió comparar la actuación de ambos grupos. Por este motivo, este resultado debe ser tomado con cautela. Pensamos que el análisis de las demás tareas de clasificación contribuirá a esclarecer este asunto.

Este primer avance de resultados procedentes de varias pruebas de la batería Nombela - la mayoría de ellas consideradas clásicas en el estudio del deterioro categorial- sugiere que es un instrumento adecuado para evaluar el deterioro semántico de los pacientes de Alzheimer. Además, hay que tener en cuenta la mejora que representa respecto a otros instrumentos similares, sobre todo, en relación al número de categorías evaluadas, a la inclusión de categorías consideradas problemáticas por la literatura y, lo que consideramos de mayor relevancia, al riguroso control de variables que influyen en la evaluación del deterioro semántico (Hodges et al., 1995; Laiacona et al., 1993; Peraita et al., 2000). Que nosotros sepamos, este es el primer instrumento que engloba tales características.

Conocer en profundidad la organización, funcionamiento y deterioro de los procesos cognitivos nos permitirá diseñar programas de intervención terapéutica eficaces para hacer frente a enfermedades que, como la EA, afectan cada vez a un mayor número de personas. Pensamos que la batería Nombela es un instrumento que será de interés para los investigadores de lengua española, en especial para los interesados en la población anciana y en los problemas que, como la demencia, se asocian a este grupo de edad.
Además de la evaluación del deterioro semántico categorial, existen otros potenciales campos de aplicación del instrumento. Por ejemplo, sus items pueden utilizarse en estudios de neuroimagen funcional, en estudios sobre el procesamiento de los objetos y en la evaluación del patrón de cambio cognitivo en estudios longitudinales (p.ej., para evaluar la efectividad de nuevos fármacos). Otros campos de aplicación, podrían ser la evaluación forense neuropsicológica, cuando es necesario conocer $-\mathrm{y}$ poder cuantificar - el deterioro semántico (p.ej., en accidentes automovilísticos, o en la incapacitación legal de pacientes con demencia), así como otras áreas de la psicopatología y psicología clínica en las que se emplean técnicas y procedimientos alternativos para la evaluación de las distintas funciones neuropsicologicas y/o cognitivas (p.ej., Lopez-Luengo y Florit, 2003; Llevaria, Prats, Vicens-Vilanova y Obiols, 2003).

\section{REFERENCIAS}

Azuma, T., Bayles, K.A:, Cruz, R.F., Tomoeda, C.K., Wood, J.A. McGeah, A. y Montgomery, E.B. (1997). Comparing the difficulty of letter, semantic, and name fluency task for normal elderly and patients with Parkinson's disease. Neuropsychology, 11, 488-497.

Barbarotto, R., Capitani, E y Laiacona, M. (2001). Living musical instruments and inanimate body parts? Neuropsychologia, 39, 406-414.

Barbarotto, R., Laiacona, M., Macchi, V y Capitani, E. (2002). Picture reality decision, semantic categories and gender. A new set of pictures, with norms and an experimental study. Neuropsychologia, 40 , 1637-1653.

Blesa, R., Pujol, M., Aguilar, M., Santacruz, P., Bertran-Serra, I., Hernández, G., Sol, J.M. y Peña-Casanova, J. (2001). Clinical validity of the 'mini-mental state for Spanish spea- 
king communities. Neuropsychologia, 39, 1150-1157.

Bright, P., Moss, H. y Tyler, L.K. (en prensa). Commentary on Keith R. Laws: «illusions of normality": A methodological critique of category-specific naming.

Brousseau, G. y Buchanan, L. (2005). Semantic category effect and emotional valence in female university students. Brain and Language, 90, 241-248.

Bunn, E.M., Tyler, L.K. y Moss H.E. (1998). Category-specific semantic deficit: The role of familiarity and property type reexamined. Neuropsychology, 12, 367-379.

Capitani, E., Laiacona, M. y Barbarotto, R. (1999). Gender affects word retrieval of certain categories in semantic fluency tasks. Cortex, 35, 273-278.

Capitani, E., Laiacona, M., Mahon, B. y Caramazza, A. (2003). What are the facts of semantic category-specific deficits? A critical review of the clinical evidence. Cognitive Neuropsychology, 20, 213-261.

Caramazza, A. y Shelton, J.R. (1998). Domainspecific knowledge systems in the brain: the animate-inanimate distinction. Journal of Cognitive Neuroscience, 10, 1-34.

Coppens, P. y Frisinger, D. (2005). Categoryspecific naming effect in non-brain-damaged individuals. Brain and Language, 94, 61-71.

Daum, I., Riesch, G., Sartori, G. y Birbaumer, N. (1996). Semantic memory impairment in Alzheimer's disease. Journal of Clinical and Experimental Neuropsychology, 18, 648-665.

Diaz, M., Sailor, K., Cheung, D. y Kuslansky, G. (2004). Category size effects in semantic and letter fluency in Alzheimer's patients. Brain and Language, 89, 108-114.

Durrant-Peatfield, M.R., Tyler, L.K., Moss, H.E. y Levy, J.P. (1997). The distinctiveness of form and function in category structure: A connectionist model. En M.G. Shafto y P. Langley (Eds.), Proceedings of the 19th Annual Conference of the Cognitive Science Society (pp. 193-198). Mahwah, NJ: Lawrence Erlbaum Associates, Inc.

Folstein, M.F., Folstein, S.E. y McHugh, P.R. (1975) "Mini-Mental State»: a practical method for grading the cognitive state of patients for the clinician. Journal of Psychiatric Research, 12, 189-198.
Funnell, E. y Sheridan, J. (1992). Categories of knowledge: unfamiliar aspects of living and nonliving things. Cognitive Neuropsychology, 9, 135-153.

Gaffan, D. y Heywood, C.A. (1993). A spurious category-specific visual agnosia for living things in normal human and nonhuman primates. Journal of Cognitive Neuroscience, $5,118-128$.

Gale, T.M., Laws, K.R. y Foley, K. (en prensa). Crowded and sparse domains in object recognition: Consequences for categorization and naming.

Garrard, P., Patterson, K., Watson, P.C. y Hodges, J.R. (1998). Category specific semantic loss in dementia of Alzheimer's type. Functional-anatomical correlations from cross-sectional analyses. Brain, 121, 633646.

Gonnerman, L.M., Andersen, E.S., Devlin, J.T., Kempler, D. y Seidenberg, M.S. (1997). Double dissociation of semantic categories in Alzheimer's disease. Brain and Language, 57, 254-279.

Hillis, A.E. y Caramazza, A. (1991). Categoryspecific naming and comprehension impairment. A double dissociation. Brain, 114, 2081-2094.

Hodges, J.R., Graham, N. y Patterson, K.A. (1995). Charting the progression in semantic dementia: Implications for the organization of semantic memory. Memory, 3, 463-496.

Laiacona, M., Barbarotto, R., Trivelli, C. y Capitani, E. Disossociazioni semantiche intercategoriali: descrizione di una batteria standrdizzata e dati normativi. Archivio di Psicologia, Neurologia e Psichiatria, 54, 209-248.

Lambon Ralph, M.A., Howard, D., Nightingale, G. y Ellis, A.W. (1998). Are living and non-living category-specific deficits causally linked to impaired perceptual or associative knowledge? Evidence from a category-specific double dissociation. $\mathrm{Neu}$ rocase, 4, 311-338.

Laws, K.R. (1999). Gender affects naming latencies for living and nonliving things: Implications for familiarity. Cortex, 35, 729-733.

Laws, K.R., Gale, T.M., Leeson, V.C., and Crawford, J.R. (2005). When is category- 
specific in Alzheimer's disease. Cortex, 41, 452-463.

López-Luengo, B., y Florit, A. (2003). Análisis del efecto de aprendizaje en tareas atencionales simples y duales: Un estudio comparativo entre personas diagnosticadas de esquizofrenia y personas sin diagnóstico psiquiátrico. Revista de Psicopatología y Psicología Clínica, 8, 211-222.

Llevaria, G., Prats, E., Vicens-Vilanova, J., y Obiols, J.E. (2003). Preferencia manual en el espectro esquizofrénico. Revista de Psicopatología y Psicología Clínica, 8, 189198.

Magnié, M.N., Besson, M., Poncet, M. y Dolisi, C. (2003) The Snodgrass and Vanderwart set revisited: Norms for object manipulability and for pictorial ambiguity of objects, Chimeric Objects and nonobjects. Journal of Clinical and Experimental Neuropsychology, 25, 521-560.

Martin, A. y Caramazza, A. (2003). Neuropsychological and neuroimaging perspectives on conceptual knowledge: An introduction. Cognitive Neuropsychology, 3-6, 195-212.

McRae, K., y Cree, G.S. (2002). Factors underlying category-specific semantic deficits. En E. M. E. Forde y G. W. Humphreys (Eds.), Category-specificity in mind and brain (pp. 211-249). East Sussex, UK: Psychology Press.

Monsch, A.U., Bondi, M.W., Butters, N., Salmon, D.P., Katzman, R. y Thal, L.J. (1992). Comparisons of verbal fluency tasks in the detection of dementia of the Alzheimer type. Archives of Neurology, 49, 12531258.

Montanes, P., Goldblum, M.C. y Boller, F. (1995). The naming impairment of living and nonliving items in Alzheimer's disease. Journal of the International Neuropsychological Society, 1, 39-48.

Montanes, P., Goldblum, M.C. y Boller, F. (1996). Classification deficits in Alzheimer's disease with special reference to living and nonliving things. Brain and Language, 54, 335-358.

Moreno, F.J. y Peraita, H. (2003). Familiarity, visual complexity and age of acquisition bias: the assessment of semantic deterioration in Alzheimer's patients. En B. Berglund y E. Borg (Eds.), Fechner Day 2003 (pp. 193-198). Stockholm: International Society for Psychophysics.

Moreno, F.J., Peraita, H., Cañamón, S. y Silva, A.I. (2004). Deterioro semántico categorial en la enfermedad de Alzheimer: un nuevo conjunto de estímulos para su evaluación [Resumen]. En Facultad de Psicología (Ed.), Evaluación neuropsicológica y de aspectos psicofisiológicos. VII European Conference on Psychological Assessment. $E A P A-2004$, (pp. 190) Málaga: Universidad de Málaga.

Moreno, F.J. y Peraita, H. (en prensa). A new set of items for evaluation of living / nonliving dissociations with norms collected from healthy elderly Spanish [Resumen]. Journal of the International Neuropsycological Society.

Moreno, F.J. y Peraita, H. (2005a). Denominación de fotografías a color de un grupo de pacientes de Alzheimer y otro de ancianos sanos. Manuscrito no publicado.

Moreno, F.J. y Peraita, H. (2005b). Un nuevo conjunto de items para la evaluación de la disociación vivo / artefacto con normas obtenidas de ancianos sanos españoles. Manuscrito enviado para su publicación.

Nebes, R.D. (1989). Semantic memory in Alzheimer's disease. Psychological Bulletin, 106, 377-394.

Nebes, R.D., Martin, D.C. y Horn, L.C. (1984). Sparing of semantic memory in Alzheimer's disease. Journal of Abnormal Psychology, 93, 321-330.

Nielsen, J.M. (1946). Agnosia, Apraxia: Their Value in Cerebral Localization, $2^{\text {nd }}$ Edition. New York: Hoeber.

Ober, B. y Shenaut, G. (1995). Semantic priming in Alzheimer's disease: Meta-analysis and theoretical evaluation. En P. Allen y T. Bashore (Eds.), Age, Differences in Word and Language Processing (pp. 247271). Ámsterdam: Elsevier.

Peraita, H, González-Labra, M.J., Sánchez Bernardos, M.L. y Galeote, M. (2000). Batería de evaluación de la memoria semántica en Alzheimer. Psicothema, 12, 192-200.

Perri, R., Carlesimo, G.A., Zannino, G.D., Mauri, M., Muolo, B., Pettenati, C. y Caltagirone, C. (2003). Intentional and automatic measures of specific-category effect in the semantic impairment of patients with 
Alzheimer's disease. Neuropsychologia, 21, 1509-1522.

Rich, J.B., Park, N.W., Dopkins, S. y Brandt, J. (2002). What do Alzheimer's disease patients know about animals? It depends on task structure and presentation format. Journal of the International Neuropsychological Society, 8, 83-94.

Sanfeliu M.C., y Fernández, A. (1996). A set of 254 Snodgrass-Vanderwart's pictures standardized for Spanish: Norms for name agreement, image agreement, familiarity and visual complexity. Behavior Research Methods, Instruments and Computers, 28, 537-555.

Shelton, J. R., Fouch, E. y Caramazza, A. (1998). The selective sparing of body-part knowledge: a case study. Neurocase, 4, 339-351.

Sheridan, J. y Humphreys, G.W. (1993). A verbal-semantic category-specific recognition impairment. Cognitive Neuropsychology, 10, 143-184.

Silveri, M.C., Cappa, A., Mariotti, P. y Puopolo, M. (2002). Naming in patients with Alzheimer's disease: Influence of age of acquisition and categorical effects. Journal of Clinical and Experimental Neruopsychology, 24, 755-764.

Snodgrass, J.G. y Vanderwart, M. (1980). A Standardized Set of 260 Pictures: Norms for Name Agreement, Image Agreement, Familiarity, and Visual Complexity. Journal of Experimental Psychology: Human Learning and memory, 6, 174-215.

Soto, P., Sebastián, M.V., García, E. y del Amo, T. (1994). Las categorías y sus normas en castellano. Madrid: Visor Distribuciones.

Stewart, F., Parkin, A.J. y Hunkin, N.M. (1992). Naming Impairments Following Recovery from Herpes Simplex Encephalitis: Category-specific? The Quarterly Journal of Experimental Psychology, 11A, 261284.

Thompson-Schill, S.L. (2003). Neuroimaging studies of semantic memory: inferring "how" from "where". Neuropsychologia, 41, 280-292.

Warrington, E.K. y McCarthy, R.A. (1987). Categories of Knowledge: Further Fractionations and a Attempted Integration. Brain, 110, 1273-1296.

Warrington, E.K. y Shallice, T. (1984). Category Specific Semantic Impairments. Brain, 107, 829-854.

Zannino, G.D., Perri, R., Carlesimo, G.A., Pasqualetti, P. y Caltagirone, C. (2002). Category-specific impairment in patients with Alzheimer's disease as a function of disease severity: a cross-sectional investigation. Neuropsychologia, 40, 2268-2279. 